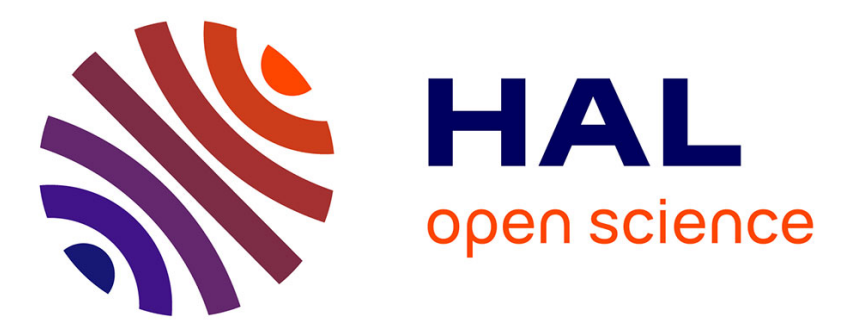

\title{
Incremental algorithm for hierarchical minimum spanning forests and saliency of watershed cuts
}

\author{
Jean Cousty, Laurent Najman
}

\section{To cite this version:}

Jean Cousty, Laurent Najman. Incremental algorithm for hierarchical minimum spanning forests and saliency of watershed cuts. 10th International Symposium on Mathematical Morphology (ISMM'11), Jul 2011, Verbania-Intra, Italy. pp.272-283， 10.1007/978-3-642-21569-8_24 . hal-00622505

\section{HAL Id: hal-00622505 https://hal.science/hal-00622505}

Submitted on 12 Sep 2011

HAL is a multi-disciplinary open access archive for the deposit and dissemination of scientific research documents, whether they are published or not. The documents may come from teaching and research institutions in France or abroad, or from public or private research centers.
L'archive ouverte pluridisciplinaire HAL, est destinée au dépôt et à la diffusion de documents scientifiques de niveau recherche, publiés ou non, émanant des établissements d'enseignement et de recherche français ou étrangers, des laboratoires publics ou privés. 


\title{
Incremental algorithm for hierarchical minimum spanning forests and saliency of watershed cuts ${ }^{\star}$
}

\author{
Jean Cousty and Laurent Najman \\ Université Paris-Est, Laboratoire d'Informatique Gaspard-Monge, A3SI, ESIEE \\ $\{j$.cousty, l.najman\}@esiee.fr
}

\begin{abstract}
We study hierarchical segmentations that are optimal in the sense of minimal spanning forests of the original image. We introduce a region-merging operation called uprooting, and we prove that optimal hierarchical segmentations are equivalent to the ones given by uprooting a watershed-cut based segmentation. Based on those theoretical results, we propose an efficient algorithm to compute such hierarchies, as well as the first saliency map algorithm compatible with the morphological filtering framework.
\end{abstract}

\section{Introduction}

We study some optimality properties of hierarchical segmentations ([1-9]) in the framework of edge-weighted graphs, where the cost of an edge is given by a dissimilarity between two points of an image. Since the pioneering work of $[10,11]$ stating an equivalence between hierarchies and minimum spanning trees (MST), a large number of hierarchical schemes rely on the construction of such a tree. Its first appearance for classification in pattern recognition dates from the seminal work of Zahn [12]. Its use for image segmentation was introduced by Morris et al. [13] in 1986 and popularized in 2004 by Felzenswalb and Huttenlocher [14]. Meyer was the first to explicitly use it in a morphological context [1]. In mathematical morphology, hierarchies of watershed regions have been proposed notably in [2-4] and recently reviewed in [7].

In this paper, we formalize, in the framework of edge-weighted graph, a fundamental hierarchical scheme proposed in morphology. This formalism allows us to prove several strong properties linking hierarchical segmentations and combinatorial optimality with respect to the original image (Th. 4 and 9). We derive from those properties the first saliency algorithm proved to be correct, establishing in particular a compatibility property with morphological filtering (Th. 11). In contrast, all the previously proposed algorithms (e.g. $[2,3,15])$ are heuristic by nature, and to date, no property on their result has been proved. With those previous algorithms, counter-intuitive results are often obtained [16].

After reminding basic notions in Section 2, hierarchies of minimum spanning forests are presented in Section 3. Then, we formalize, in Section 4, a fundamental

* This work received funding from the Agence Nationale de la Recherche, contract ANR-2010-BLAN-0205-01. 
operation called uprooting that merges a marked region with one of its neighbors linked by the cheapest cost. When applied sequentially on the weighted graph of neighboring regions, the uprootings build a MST of this neighboring graph. Intuitively, one can see that, if one starts from a minimum spanning forest (MSF) rooted in the minima of the image (or, equivalently, from a watershed cut [17]), then one builds a hierarchy of MSFs of the original image itself, the last uprooting step yielding an MST of this original image. More surprisingly, Th. 4 establishes that the two processes are equivalent. Hence, any MST of the original image can be built from an uprooting sequence on a watershed cut. Thus, watershed cuts are the only family of watersheds that allows us to build hierarchical segmentations that are optimal with respect to the original image, in the sense that they preserve the MST of the original image. Based on those results, we give a detailed description of a fast uprooting algorithm that allows MSF hierarchies to be computed. The time-complexity of this algorithm is analyzed. Then, in Section 5, a linear-time algorithm is proposed to compute saliency maps from MSF hierarchies. The correctness of this algorithm is established by Theorem 9 . Finally, in Section 6, the compatibility between the MSF hierarchies and a morphological filtering is established by an equivalence result (Theorem 11).

\section{Basic Notions}

We define a (undirected) graph as a pair $X=(V, E)$ where $V$ is a finite set and $E$ is composed of unordered pairs of $V$, i.e., $E$ is a subset of $\{\{x, y\} \subseteq V \mid x \neq y\}$. If $X=(V, E)$ is a graph, each element of $V$ is called a vertex or a point of $X$, and each element of $E$ is called an edge of $X$.

Let $X$ be a graph, the vertex set and edge set of $X$ are denoted by $V(X)$ and $E(X)$ respectively. The graph $X$ is nontrivial whenever $E(X) \neq \emptyset$, and it is nonempty whenever $V(X) \neq \emptyset$.

Let $X$ and $Y$ be two graphs. If $V(Y) \subseteq V(X)$ and $E(Y) \subseteq E(X)$, we say that $Y$ is a subgraph of $X$ and we write $Y \sqsubseteq X$. Let $\mathcal{S}$ be a set of graphs. The supremum (resp. infimum) of $\mathcal{S}$, denoted by $\sqcup \mathcal{S}$ (resp. $\sqcap \mathcal{S}$ ), is the graph whose vertex set and edge set are the unions (resp. intersections) of the vertex sets and of the edge sets of the graphs in $\mathcal{S}: \sqcup \mathcal{S}=(\cup\{V(X) \mid X \in \mathcal{S}\}, \cup\{E(X) \mid X \in \mathcal{S}\})$ (resp. $\sqcap \mathcal{S}=(\cap\{V(X) \mid X \in \mathcal{S}\}, \cap\{E(X) \mid X \in \mathcal{S}\}))$. We also write $X \sqcup Y$ and $X \sqcap Y$ for respectively $\sqcup\{X, Y\}$ and $\sqcap\{X, Y\}$.

Let $X$ be a graph. The graph $X$ is connected if it cannot be partitioned into two nonempty graphs, i.e., for any two nonempty graphs $Y$ and $Z$, if $Y \sqcup Z=X$, then the graph $Y \sqcap Z$ is necessarily nonempty. Let $Y$ be a graph. We say that $Y$ is a connected component of $X$, or simply a component of $X$, if $Y$ is a subgraph of $X$ that is connected and that is maximal for this property, i.e., for any connected graph $Z$, if $Y \sqsubseteq Z \sqsubseteq X$, then we have necessarily $Z=Y$. The set of all connected components of $X$ is denoted by $\mathcal{C C}(X)$.

Important notation. Throughout this paper $G=(V, E)$ denotes a nontrivial connected graph. 
If $S$ is a subset of $V$ (resp. $E$ ), we denote by $\bar{S}$ the complementary set of $S$ in $V$ (resp. E), i.e., $\bar{S}=V \backslash S$ (resp. $\bar{S}=E \backslash S)$.

Let $S \subseteq E$, we denote by $\delta^{\bullet}(S)$ the set of all vertices in $V$ that belong to an edge in $S$. Remark that $\left(\delta^{\bullet}(S), S\right)$ is a graph (see [18] for morphological properties of $\delta^{\bullet}$ ). This graph $\left(\delta^{\bullet}(S), S\right)$ is called the graph induced by $S$.

In the following, the sets of integers and real numbers are denoted by $\mathbb{Z}$ and $\mathbb{R}$ respectively. Let $i, j \in \mathbb{Z}$, we denote by $[i, j]$ the set $\{k \in \mathbb{Z} \mid i \leq k \leq j\}$.

We denote by $\mathcal{F}$ the set of all maps from $E$ into $\mathbb{R}$, and we say that any map in $\mathcal{F}$ weights the edges of $G$.

Let $F \in \mathcal{F}$. If $u$ is an edge of $G$, the number $F(u)$ is the altitude or weight of $u$. Let $k \in \mathbb{R}$, we denote by $F[k]$ the set of edges of $G$ whose weight (for $F$ ) is less than or equal to $k: F[k]=\{u \in E \mid F(u) \leq k\}$. The set of edges $F[k]$ is called the (lower) cross section of $F$ at level $k$.

Let $F \in \mathcal{F}$, and let $X \sqsubseteq G$. We say that $X$ is a connected component of $F$, or simply a component of $F$, if there exists an element $k \in \mathbb{R}$ such that $X$ is a component of the graph induced by $F[k]$. We denote by $\mathcal{C C}(F)$ the set of all components of $F$. The set $\mathcal{C C}(F)$ is closely related to the component tree [19, $20]$ of $F$, a tree widely used for filtering.

Let $F \in \mathcal{F}$, and let $X \sqsubseteq G$. The graph $X$ is a minimum of $F$ if $X$ is a component of $F$ that does not strictly contains any component of $F$, i.e., for any $Y \in \mathcal{C C}(F)$, if $Y \sqsubseteq X$, then we have necessarily $Y=X$. We denote by $\mathcal{M}_{F}$ the set made of the minima of $F$, and by $M_{F}$ its supremum: $M_{F}=\sqcup \mathcal{M}_{F}$. This notion of minima, as well as further presented notions, is illustrated in Fig. 1

Important notation. In the sequel of this paper, $F$ denotes an element of $\mathcal{F}$. Therefore the pair $(G, F)$ is called an edge-weighted graph.

\section{Minimum spanning forests hierarchies}

This section first presents the minimum spanning forests rooted in subgraphs of $G$. This notion of a forest, which is useful for (seeded) image segmentation (see e.g. $[17,21,22])$, is known to be equivalent to the one of minimum spanning tree studied in combinatorial optimization. Then, hierarchies of minimum spanning forests are introduced. Each such hierarchy induces a hierarchy of partitions on $V$, which is thus optimal in the sense of rooted minimum spanning forests.

Let $X \sqsubseteq G$. The weight of $X$ (for $F$ ), denoted by $F(X)$, is the sum of the weights of the edges in $E(X): F(X)=\sum_{u \in E(X)} F(u)$. Let $V^{\prime} \subseteq V$, we say that $X$ is spanning for $V^{\prime}$ if $V(X)=V^{\prime}$.

Definition 1 (rooted MSF) Let $X$ and $Y$ be two nonempty subgraphs of $G$.

We say that $Y$ is rooted in $X$ if $V(X) \subseteq V(Y)$ and if the vertex set of any component of $Y$ contains the vertex set of exactly one component of $X$.

We say that $Y$ is a minimum spanning forest (MSF) rooted in $X$ (with respect to $F$ ) if:

1. $Y$ is spanning for $V$;

2. $Y$ is rooted in $X$; and 


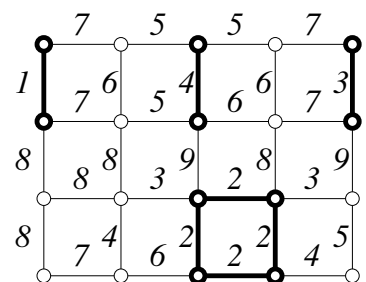

(a)

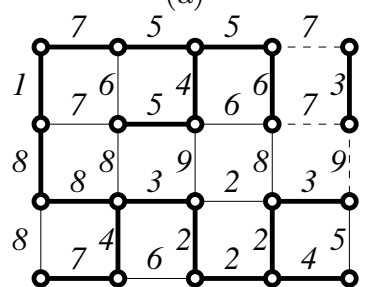

(d)

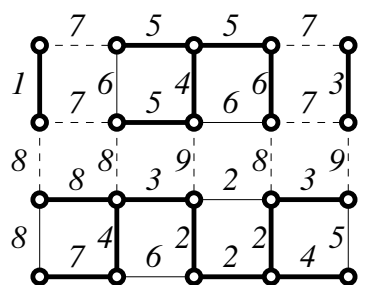

(b)

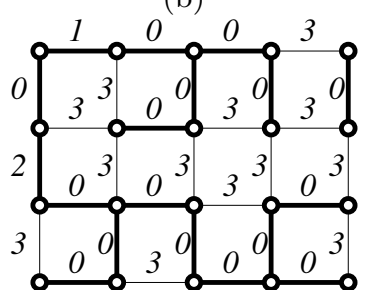

(e)

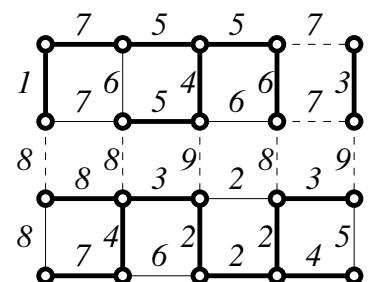

(c)

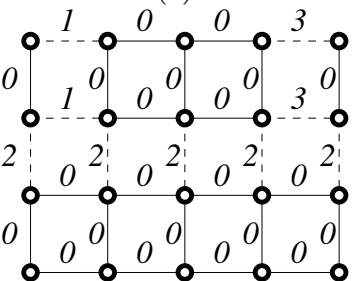

(f)

Fig. 1. (a): A graph $G$ and a map $F$ whose minima are depicted in bold. (b): A graph $X_{0} \in M S F\left(M_{F}\right)$ represented in bold and its induced cut $C_{0}=C\left(X_{0}\right)$ represented by dashed edges. (c,d): two bold graphs called respectively $X_{1}$ and $X_{2}$ such that $\mathcal{T}=\left\langle X_{0}, X_{1}, X_{2}\right\rangle$ is both an MSF hierarchy for and an uprooting by $\left\langle M_{1}, M_{2}\right\rangle$ (where $M_{i}$ is the minimum of $F$ whose altitude is $i$ ); their induced cuts $C_{1}=C\left(X_{1}\right)$ and $C_{2}=C\left(X_{2}\right)$ are represented by dashed edges. (e): The indicator of the uprooting $\mathcal{U}$. (f): The saliency map of the MSF cut hierarchy $\left\langle C_{0}, C_{1}, C_{2}\right\rangle$.

3. the weight of $Y$ is less than or equal to the weight of any graph $Z$ satisfying (1) and (2) (i.e., $Z$ is both spanning for $V$ and rooted in $X$ ).

The set of all minimum spanning forests rooted in $X$ is denoted by $M S F(X)$.

The above definition of rooted MSFs, which is illustrated in Fig. 1b, allows the usual notions of graph theory based on trees and forests to be recovered. In particular, if $x$ is a vertex of $V$, it can be seen that any element in $M S F((\{x\}, \emptyset))$ is a minimum spanning tree of $(G, F)$, and that, conversely, any minimum spanning tree of $(G, F)$ belongs to $M S F((\{x\}, \emptyset))$. In the following, by convention, this remarkable set, which is made of all minimum spanning trees of $(G, F)$, will also be denoted by $M S F((\emptyset, \emptyset))$.

A possible definition for watershed, called watershed cuts, follows the drop of water principle. In [17], we have proved the equivalence between MSF rooted in the set of minima and watershed cuts. In practice, watersheds from markers are often computed, and subsets of minima of the original edge-weighted graph constitute robust markers [23]. The next definition, illustrated in Figs. 1b, c, and $\mathrm{d}$, presents a notion of hierarchy of MSFs rooted in such subsets.

Definition 2 (MSF hierarchy) Let $\mathcal{S}=\left\langle M_{1}, \ldots, M_{\ell}\right\rangle$ be a sequence of pairwise distinct minima of $F$ and let $\mathcal{T}=\left\langle X_{0}, \ldots X_{\ell}\right\rangle$ be a sequence of subgraphs of $G$. We say that $\mathcal{T}$ is an MSF hierarchy for $\mathcal{S}$ if: 
1. for any $i \in[0, \ell]$, the graph $X_{i}$ is an $M S F$ rooted in $\sqcup\left[\mathcal{M}_{F} \backslash\left\{M_{j} \mid j \in[1, i]\right\}\right]$; and

2. for any $i \in[1, \ell]$, we have $X_{i-1} \sqsubseteq X_{i}$.

\section{Uprootings and MSF hierarchies}

In this section, we formalize a simple transformation, called uprooting, that allows a forest $X$ rooted in a graph $M$ to be incrementally transformed into a forest $Y$ rooted in a graph $M^{\prime}$ obtained by removing some components of $M$. Through an equivalence theorem, we establish an important link between the uprooting transform and the MSF hierarchies. This result allows efficient algorithms for computing MSF hierarchies to be considered.

Let $X$ be a subgraph of $G$ that is spanning for $V$, and let $x \in V$. We denote by $C C_{x}(X)$ the component of $X$ whose vertex set contains $x$. Let $V^{\prime} \subseteq V$, we set $C C_{V^{\prime}}(X)=\sqcup\left\{C C_{x}(X) \mid x \in V^{\prime}\right\}$.

Let $X \subseteq G$, and let $u=\{x, y\} \in E$. The edge $u$ is outgoing from $X$ if $u$ is made of a vertex in $V(X)$ and of a vertex in $\overline{V(X)}$. In the following, by abuse of notation, we write $X \sqcup\{u\}$ for the supremum of $X$ and the graph induced by $\{u\}: X \sqcup\{u\}=(V(X) \cup u, E(X) \cup\{u\})$.

Let $X, Y$, and $M$ be three subgraphs of $G$ such that $X$ is spanning for $V$ and such that $X \neq Y$. We say that $Y$ is an elementary uprooting of $X$ by $M$ if there exists an edge $u$ of minimum weight among the edges outgoing from $C C_{V(M)}(X)$ such that $Y=X \sqcup\{u\}$. We also say that $Y$ is an elementary uprooting of $X$ by $M$ if $Y=X$ and if there is no edge outgoing from $C C_{V(M)}(X)$.

Definition 3 Let $\mathcal{S}=\left\langle M_{1}, \ldots, M_{\ell}\right\rangle$ be a sequence of pairwise distinct minima of $F$. An uprooting by $\mathcal{S}$ is a sequence $\left\langle X_{0}, \ldots, X_{\ell}\right\rangle$ of graphs such that:

1. $X_{0} \in M S F\left(M_{F}\right)$; and

2. $X_{i}$ is an elementary uprooting of $X_{i-1}$ by $M_{i}$, for any $i \in[1, \ell]$.

The following theorem, one of the main results of this paper, states an equivalence property between MSF hierarchy and uprooting from a subset of minima of the original graph.

Theorem 4 Let $\mathcal{S}=\left\langle M_{1}, \ldots, M_{\ell}\right\rangle$ be a sequence of pairwise distinct minima of $F$, and let $\mathcal{T}=\left\langle X_{0}, \ldots X_{\ell}\right\rangle$ be a sequence of subgraphs of $G$. The sequence $\mathcal{T}$ is an MSF hierarchy for $\mathcal{S}$ if and only if the sequence $\mathcal{T}$ is an uprooting by $\mathcal{S}$.

Any MSF hierarchy can be represented by a unique edge-weighed graph such that a given threshold of this graph provides the associated level of the hierarchy. More formally, let $\mathcal{S}=\left\langle M_{1}, \ldots, M_{\ell}\right\rangle$ be a sequence of pairwise distinct minima of $F$, and let $\mathcal{T}=\left\langle X_{0}, \ldots, X_{\ell}\right\rangle$ be an uprooting by $\mathcal{S}$. The indicator of $\mathcal{T}$, denoted by $I_{\mathcal{T}}$, is the map from $E$ into $[0, \ell+1]$ defined by:

- $I_{\mathcal{T}}(u)=\min \left\{i \in[0, \ell] \mid u \in E\left(X_{i}\right)\right\}$, for any $u \in E\left(X_{\ell}\right)$; and

- $I_{\mathcal{T}}(u)=\ell+1$, for any $u \in E \backslash E\left(X_{\ell}\right)$.

The notion of indicator of an uprooting is illustrated in Fig. 1e. 
Any uprooting has a unique indicator, and any two distinct uprootings have distinct indicators. Hence, the indicator of an uprooting is sufficient to recover all the elements of the uprooting: if $\mathcal{T}=\left\langle X_{0}, \ldots, X_{\ell}\right\rangle$ is an uprooting by a sequence $\mathcal{S}=\left\langle M_{1}, \ldots, M_{\ell}\right\rangle$ of minima of $F$, then $X_{i}$ is the graph induced by the cross-section of $I_{\mathcal{T}}$ at level $i,\left(i . e ., X_{i}=\left(\delta^{\bullet}\left(I_{\mathcal{T}}[i]\right), I_{\mathcal{T}}[i]\right)\right.$.

We are now ready to describe the uprooting algorithm, which by Theorem 4 allows the computation of MSF hierarchies. It inputs a sequence $\mathcal{S}=$ $\left\langle M_{1}, \ldots, M_{\ell}\right\rangle$ of minima of $F$ and outputs the indicator $I_{\mathcal{T}}$ of an uprooting $\mathcal{T}=$ $\left\langle X_{0}, \ldots, X_{\ell}\right\rangle$ by $\mathcal{S}$. From a high level point of view, the algorithm can be sketched, once a minimum spanning forest $X$ relative to $M$ has been computed, by iterations of the following three region merging steps:

i Find the component $C$ of $X$ that contains $M_{i}$ the next element in $\mathcal{S}$.

ii Find a component $C^{\prime}$ of $X$ linked to $C$ by an edge $v$ of minimum weight iii Merge the two components $C$ and $C^{\prime}$

In order to ease the reading of the algorithm, let us first present the two main data structures that are used.

The structure $\mathcal{C}$ is a collection of disjoint subsets of $V$. Each set $C$ in $\mathcal{C}$ is represented by a unique element $x$ of $C$, called the canonical element of $C$. In the following, $x$ and $y$ denote two distinct elements of $V$. The collection $\mathcal{C}$ is managed by three operations:

- MakeSet $(x)$ : add the set $\{x\}$ to the $\operatorname{collection} \mathcal{C}$, provided that the element $x$ does not already belong to a set in $\mathcal{C}$.

- Find $(x)$ : returns the canonical element of the set in $\mathcal{C}$ that contains $x$.

- $\operatorname{Link}(x, y)$ : let $C_{x}$ and $C_{y}$ denote two sets in $\mathcal{C}$ whose canonical elements are $x$ and $y$ respectively. Both sets are removed from $\mathcal{C}$, their union $C=$ $C_{x} \cup C_{y}$ is added to $\mathcal{C}$ and a canonical element for $C$ is selected and returned.

The structure $\mathcal{L}$ is a collection of $n=|V|$ lists: $\mathcal{L}\left[x_{1}\right], \ldots, \mathcal{L}\left[x_{n}\right]$, with $V=$ $\left\{x_{1}, \ldots, x_{n}\right\}$. Each element of these lists is a triplet $(x, u, w)$ such that $x$ is a vertex of $G, u$ is an edge of $G$, and $w$ is an element in $\mathbb{R}$, called the weight of the triplet. In the following, $x$ and $y$ denote two distinct elements of $V, u$ denotes an edge in $E$, and $w$ an element in $\mathbb{R}$. The collection $\mathcal{L}$ is managed by four operations:

- MakeList(): returns an empty list.

- $\operatorname{Insert}_{x}(y, u, w)$ : adds the triplet $(y, u, w)$ to the list $\mathcal{L}[x]$;

- DeleteMin ${ }_{x}()$ returns and removes from $\mathcal{L}[x]$ a triplet of minimum weight.

- $\operatorname{Meld}(x, y):$ returns the list of the triplets of $\mathcal{L}[x]$ and of $\mathcal{L}[y]$ and suppresses the lists $\mathcal{L}[x]$ and $\mathcal{L}[y]$.

When the algorithm terminates the map $I$ is the indicator of an uprooting by the input sequence $\mathcal{S}$. Moreover, using Tarjan's union find [24] and Fredman and Tarjan's Fibonnacci heap [25] algorithms to manage the collections $\mathcal{C}$ and $\mathcal{L}$, the overall complexity of the algorithm is $O(|V| \times \alpha(|V|,|V|)+|E| \times \alpha(|E|,|V|)+$ $|E| \log |E|)$, where $\alpha$ is a function which grows very slowly: for all practical purposes $\alpha(m, n)$ is never greater than four. In other words, the complexity of the algorithm is quasi $O(|V|+|E| \log |E|)$ in the sense of Tarjan's union find. 


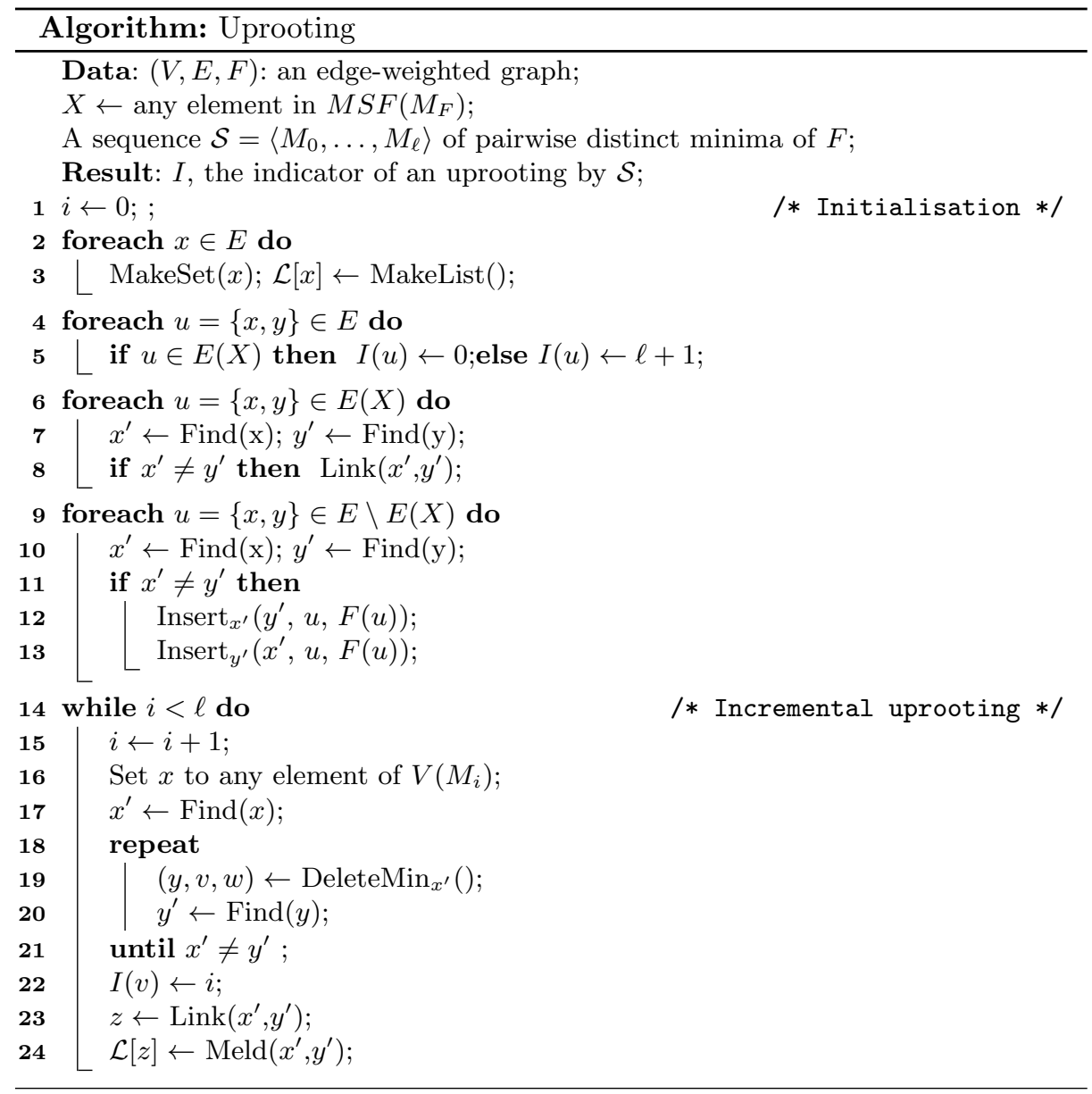

\section{MSF cut hierarchy, saliency and connection value}

Until now, we have dealt with regions (components of forests). Let us now study their "dual", that represents borders between regions and that are called cuts.

Let $X$ be a subgraph of $G$ that is spanning for $V$. The (graph) cut induced by $X$, denoted by $C(X)$, is the set of all edges of $G$ made of vertices of two distinct components of $X$, i.e. $C(X)=\left\{\{x, y\} \in V \mid C C_{x}(X) \neq C C_{y}(X)\right\}$.

Definition 5 (MSF cut hierarchy) Let $X$ be a subgraph of $G$, and let $C \subseteq E$. The set $C$ is called an MSF cut for $X$ (with respect to $F$ ) if there exists an $M S F Y$ rooted in $X$, such that $C$ is the cut induced by $Y$.

Let $\mathcal{S}=\left\langle M_{1}, \ldots, M_{\ell}\right\rangle$ be a sequence of pairwise distinct minima of $F$ and let $\mathcal{T}=\left\langle C_{0}, \ldots C_{\ell}\right\rangle$ be a sequence of subsets of $E$. We say that $\mathcal{T}$ is an MSF cut hierarchy for $\mathcal{S}$ if: 
1. for any $i \in[0, \ell]$, the set $C_{i}$ is an $M S F$ cut for $\sqcup\left[\mathcal{M}_{F} \backslash\left\{M_{j} \mid j \in[1, i]\right\}\right]$; and 2. for any $i \in[1, \ell]$, we have $C_{i} \subseteq C_{i-1}$.

The following result asserts that there is indeed an equivalence relation between MSF hierarchies and MSF cut hierarchies.

Property 6 Let $\mathcal{S}=\left\langle M_{1}, \ldots, M_{\ell}\right\rangle$ be a sequence of pairwise distinct minima of $F$ and let $\mathcal{T}=\left\langle C_{0}, \ldots C_{\ell}\right\rangle$ be a sequence of subsets of $E$. The sequence $\mathcal{T}$ is an MSF cut hierarchy if and only if there exists an MSF hierarchy $\left\langle X_{0}, \ldots, X_{\ell}\right\rangle$ such that $C\left(X_{i}\right)=C_{i}$, for any $i \in[0, \ell]$.

The hierarchies presented above are hierarchical edge-segmentations as defined in [9]. Therefore, the MSF cuts belonging to a hierarchy can be "stacked" to form a map that equivalently represents this hierarchy. Intuitively, such a map, called a saliency map, weights the cuts with their "level of disappearance" in the hierarchy. Hence, they are convenient for visualizing hierarchies.

Definition 7 (saliency map) Let $\mathcal{S}=\left\langle M_{1}, \ldots, M_{\ell}\right\rangle$ be a sequence of minima of $F$ and let $\mathcal{T}=\left\langle C_{0}, \ldots C_{\ell}\right\rangle$ be an $M S F$ cuts hierarchy for $\mathcal{S}$. The saliency map of $\mathcal{T}$, denoted by $S_{\mathcal{T}}$, is the map from $E$ into $[0, \ell+1]$ defined by:

1. $S_{\mathcal{T}}(u)=\min \left\{i \in[0, \ell] \mid u \in \overline{C_{i}}\right\}$, for any $u \in E \backslash C_{\ell}$; and

2. $S_{\mathcal{T}}(u)=\ell+1$, for any $u \in C_{\ell}$.

An illustration of the notion of saliency map is given in Fig. 1f.

Let $H$ be any map and let $k \in \mathbb{R}$, the set $\overline{H[k]}$ of the edges of $G$ whose weight (for $H$ ) is greater than $k$ is called the upper cross section of $H$ at level $k$.

Let $\mathcal{T}=\left\langle C_{0}, \ldots, C_{\ell}\right\rangle$ be an MSF cut hierarchy for a sequence $\mathcal{S}$ of minima of $F$, and let $H$ be a map from $E$ into $[0, \ell+1]$. It can be seen that $H=S_{\mathcal{T}}$ if and only if, for any $i \in[0, \ell]$, the set $C_{i}$ is the upper cross-section of $H$ at level $i$. Thus, the saliency map $S_{\mathcal{T}}$ is an equivalent representation of the hierarchy $\mathcal{T}$.

The altitudes of the passes between minima of the image play a fundamental role in morphological filtering. In our framework, pass altitudes are called connection values. In the following, we show their importance for computing a saliency map from the indicator of an uprooting.

Let $H \in \mathcal{F}$ and let $X \subseteq G$. The altitude of $X$ for $H$, denoted by $\check{H}(X)$, is the maximum value of an edge of $X$.

Definition 8 (connection value) Let $H \in \mathcal{F}$, and let $x$ and $y$ be two points of $G$. The connection value between $x$ and $y$ for $H$ (in $G$ ) is the value $\Upsilon_{H}(x, y)=$ $\min \{\check{H}(X) \mid X \in \mathcal{C C}(F)$ and $\{x, y\} \subseteq V(X)\}$.

The connection value, for any map $H$, between any two points can be computed in constant time (see [26]) from the (min) component tree $(\mathcal{C C}(H)$, $)$ of $H$, provided a linear time preprocessing (see [27]). In other words, once the component tree is built (see [20] for a quasi linear time algorithm) and preprocessed (in linear time), computing the connection value between any arbitrary pair of points can be done in constant time.

Hierarchical edge segmentations are in bijection with ultrametric watersheds [9]. The following theorem provides an analog result for MSF cut hierarchies. 
Theorem 9 Let $\mathcal{S}=\left\langle M_{1}, \ldots, M_{\ell}\right\rangle$ be a sequence of pairwise distinct minima of $F$, and let $H$ be a map from $E$ into $[0, \ell+1]$. The map $H$ is the saliency map of an MSF cut hierarchy for $\mathcal{S}$ if and only if there exists an uprooting $\mathcal{T}$ by $\mathcal{S}$ such that, for any $u=\{x, y\}, F(u)=\Upsilon_{I_{\mathcal{T}}}(x, y)$.

Hence, in order to know the value of an edge in a saliency map it is sufficient to know the connection value between the two points linked by this edge for the indicator of an uprooting by $\mathcal{S}$. Thus, knowing the indicator of an uprooting by $\mathcal{S}$ and its component tree, a saliency map can be computed in linear time, i.e., one constant time operation per edge of $G$.

Figs. 2b,c, and d illustrates the use of this algorithm on the image of Fig. 2a. The underlying graph is the one induced by the 4-adjacency relation whose edges are weighted by a simple color gradient (maximum, over the RGB channels, of the absolute differences of pixel values). The minima are ordered thanks to extinction values [28] related to dynamics, volume and color consistency, leading to sequences $\mathcal{S}_{1}, \mathcal{S}_{2}$, and $\mathcal{S}_{3}$ of minima. The saliency maps of MSF hierarchies for $\mathcal{S}_{1}, \mathcal{S}_{2}$, and $\mathcal{S}_{3}$ are rendered (up to an anamorphosis) in sub-figures b,c, and d, respectively. Figs. 3 illustrates the use of the algorithm to segment a surface (i.e., a mesh) embedded in the 3D Euclidean space. The vertex set of the considered graph is the set of triangles of the mesh, and its edge set is composed by the pair of triangles that share a common side. The edges of this graph are weighted by map which behaves like the inverse of the mean curvature of the surface (see [29]). The minima are ordered thanks to volume extinction values and the saliency maps of the induced MSF hierarchies is rendered up to an anamorphosis.

\section{Hierarchy by geodesic reconstructions}

A desirable compatibility property in the context of morphological filtering is that any threshold of a saliency map is a watershed of the geodesic reconstruction of the original map by the corresponding markers. Theorem 11 below shows that the results of our algorithm do satisfy such a compatibility property. It has to be noted that, in other frameworks (e.g., node-weighted graphs), such a property is in general not guaranteed [16].

Let $x \in V$. We denote by $\mathcal{C C}_{x}(F)$ the set of all components of $F$ whose vertex sets contain $x$. Let $V^{\prime} \subseteq V$, we set $\mathcal{C C}_{V^{\prime}}(F)=\cup\left\{\mathcal{C C}_{x}(F) \mid x \in V^{\prime}\right\}$.

Let $V^{\prime} \subseteq V$. The geodesic reconstruction of $F$ by $V^{\prime}$ is the map $F^{\prime}$ such that, for any edge $u$ in $E$, the value $F^{\prime}(u)$ is the minimum altitude of a component in $\mathcal{C C}_{V^{\prime}}(F)$ that contains $u: F^{\prime}(u)=\min \left\{\check{F}(X) \mid X \in \mathcal{C C}_{V^{\prime}}(F)\right\}$.

Watershed from markers are classically defined through geodesic reconstruction (also called swamping or flooding). It is shown in [30] that reconstruction plus MSF cut can be replaced by an MSF cut relative to the initial map.

Property 10 (from Theorem 6.3 in [30]) Let $V^{\prime} \subseteq V$, let $F^{\prime}$ be the geodesic reconstruction of $F$ by $V^{\prime}$, and let $C$ be a subset of $E$. If $C$ is an $M S F$ cut for $M_{H}$ with respect to $F$, then $C$ is an MSF cut for $M_{H}$ with respect to $F^{\prime}$. 


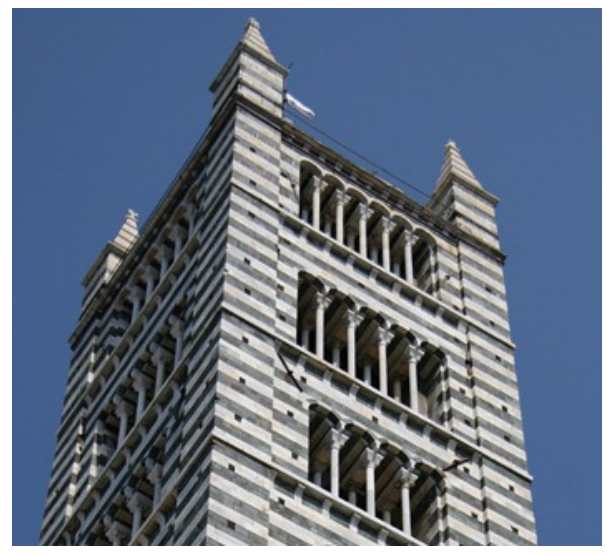

(a)

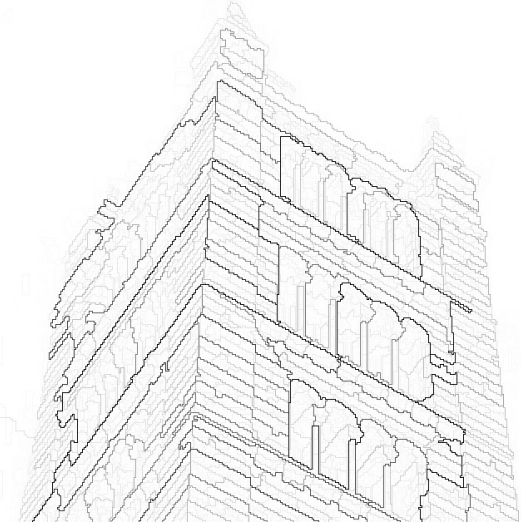

(c)

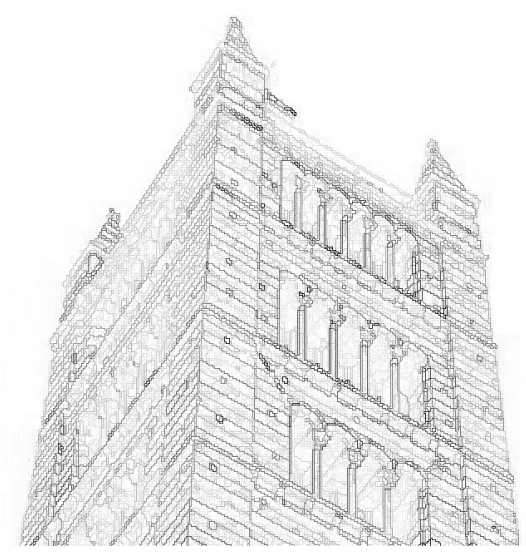

(b)

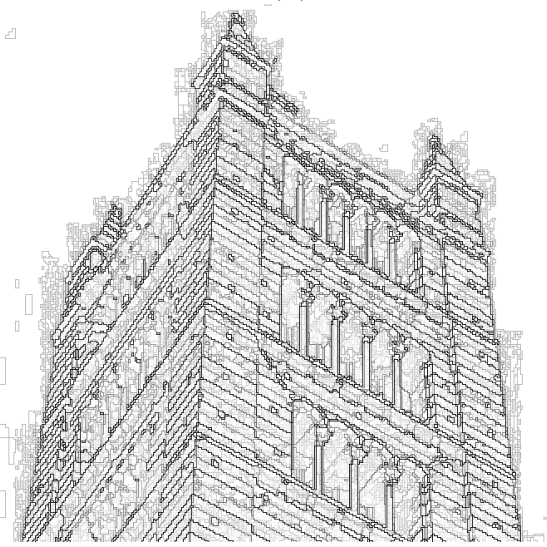

(d)

Fig. 2. Illustration of saliencies of watershed cuts (original picture (a) from koakoo: http://blog.photos-libres.fr/).

The converse of Prop. 10 is, in general, not true. In fact, as long as a sequence of nested partitions (i.e., a hierarchy) is involved, the MSF cuts of reconstructed maps and the MSF cuts of the original maps are equivalent.

Theorem 11 (Compatibility) Let $\mathcal{S}=\left\langle M_{1}, \ldots, M_{\ell}\right\rangle$ be a sequence of pairwise distinct minima of $F$. Let $G_{i}$ denote $\sqcup\left[\mathcal{M}_{F} \backslash\left\{M_{j} \mid j \in[1, i]\right\}\right]$, and let $F_{i}$ be the geodesic reconstruction of $F$ by $V\left(G_{i}\right)$, for any $i \in[0, \ell]$. Let $\mathcal{T}=\left\langle C_{0}, \ldots, C_{\ell}\right\rangle$ be a sequence of subsets of $E$ such that $C_{i} \subseteq C_{i-1}$ for any $i \in[1, \ell]$. Then, the two following propositions are equivalent:

1. for any $i \in[0, \ell]$, the set $C_{i}$ is an $M S F$ cut for $G_{i}$ with respect to $F_{i}$; and

2. for any $i \in[0, \ell]$, the set $C_{i}$ is an MSF cut for $G_{i}$ with respect to $F$. 


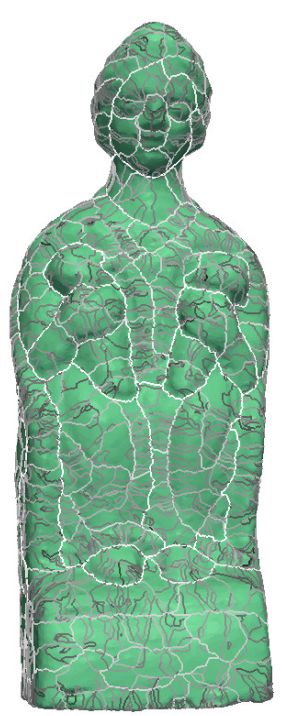

(a)

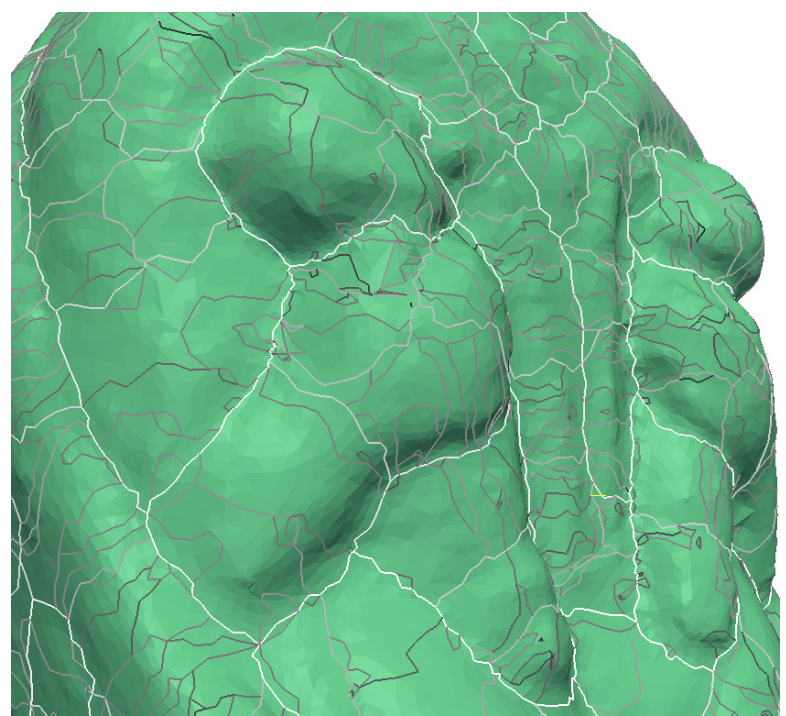

(b): zoom on a part of (a)

Fig. 3. Illustration of saliencies of watershed cuts on a mesh provided by the French Museum Center for Research and Restoration (C2RMF, Le Louvre, Paris).

\section{Conclusion and perspective}

In this paper, a classical morphological scheme for building hierarchical segmentation is formalized. This formalism leads us to establish strong properties linking hierarchical segmentations and combinatorial optimality in terms of minimum spanning forests of the original image. Recent work of Couprie et $a l$. [22] link some schemes based on minimum spanning forests to global energy minimization. Hence, a promising perspective is the investigation of hierarchical schemes defined through energy minimization such as the one presented in [5].

The source code of the algorithms presented in this paper is available at:

$$
\text { http://www.esiee.fr/ info/sm }
$$

\section{References}

1. Meyer, F.: Minimum spanning forests for morphological segmentation. In: ISMM 94. (September 1994) 77-84

2. Beucher, S.: Watershed, hierarchical segmentation and waterfall algorithm. In: ISMM 94. (1994) 69-76

3. Najman, L., Schmitt, M.: Geodesic saliency of watershed contours and hierarchical segmentation. PAMI 18(12) (December 1996) 1163-1173

4. Meyer, F.: The dynamics of minima and contours. In: ISMM. (1996) 329-336

5. Guigues, L., Cocquerez, J.P., Men, H.L.: Scale-sets image analysis. IJCV 68(3) (2006) 289-317 
6. Arbeláez, P.A., Cohen, L.D.: A metric approach to vector-valued image segmentation. IJCV 69(1) (2006) 119-126

7. Meyer, F., Najman, L.: Segmentation, minimum spanning tree and hierarchies. In: Mathematical Morphology. ISTE-Wiley (2010) 229-261

8. Cousty, J., Najman, L., Serra, J.: Raising in watershed lattices. In: 15th IEEE ICIP'08. (2008) 2196-2199

9. Najman, L.: On the equivalence between hierarchical segmentations and ultrametric watersheds. JMIV 40(3) (July 2011) 231-247 to appear.

10. Jardine, N., Sibson, R.: Mathematical taxonomy. Wiley (1971)

11. Gower, J., Ross, G.: Minimum spanning tree and single linkage cluster analysis. Appl. Stats. 18 (1969) 54-64

12. Zahn, C.: Graph-theoretical methods for detecting and descibing gestalt clusters. IEEE Transactions on Computers C-20(1) (1971) 99-112

13. Morris, O.J., Lee, M.d.J., Constantinides, A.G.: Graph theory for image analysis: an approach based on the shortest spanning tree. IEE proc. on communications, radar and signal 133(2) (1986) 146-152

14. Felzenszwalb, P., Huttenlocher, D.: Efficient graph-based image segmentation. International Journal of Computer Vision 59 (2004) 167-181

15. Marcotegui, B., Beucher, S.: Fast implementation of waterfall based on graphs. In: ISMM 2005. (2005) 177-186

16. Najman, L., Couprie, M., Bertrand, G.: Watersheds, mosaics and the emergence paradigm. DAM 147(2-3) (April 2005) 301-324

17. Cousty, J., Bertrand, G., Najman, L., Couprie, M.: Watershed Cuts: Minimum Spanning Forests and the Drop of Water Principle. PAMI 31(8) (2009) 1362-1374

18. Cousty, J., Najman, L., Serra, J.: Some morphological operators in graph spaces. In Springer, ed.: ISMM 09. Number 5720 in LNCS (2009) 149-160

19. Salembier, P., Oliveras, A., Garrido, L.: Anti-extensive connected operators for image and sequence processing. IEEE TIP 7(4) (April 1998) 555-570

20. Najman, L., Couprie, M.: Building the component tree in quasi-linear time. IEEE TIP 15(11) (2006) 3531-3539

21. Cousty, J., Bertrand, G., Najman, L., Couprie, M.: Watershed cuts: thinnings, shortest-path forests and topological watersheds. PAMI 32(5) (2010) 925-939

22. Couprie, C., Grady, L., Najman, L., Talbot, H.: Power Watersheds: A Unifying Graph Based Optimization Framework. PAMI (2010) To appear.

23. Audigier, R., Lotufo, R.: Seed-relative segmentation robustness of watershed and fuzzy connectedness approaches. In: IEEE SIBGRAPI'07. (2007) $61-70$

24. Tarjan, R.E.: Efficiency of a good but not linear set union algorithm. J. ACM 22 (1975) 215-225

25. Fredman, M.L., Tarjan, R.E.: Fibonacci heaps and their uses in improved network optimization algorithms. J. ACM 34 (1987) 596-615

26. Couprie, M., Najman, L., Bertrand, G.: Quasi-linear algorithms for the topological watershed. JMIV 22(2-3) (2005) 231-249

27. Bender, M., Farach-Colton, M.: The LCA problem revisited. In: Latin American Theoretical INformatics. (2000) 88-94

28. Vachier, C., Meyer, F.: Extinction value: a new measurement of persistence. In: IEEE Workshop on Nonlinear Signal and Image Processing. (1995) 254-257

29. Philipp-Foliguet, S., Jordan, M., Najman, L., Cousty, J.: Artwork 3D Model Database Indexing and Classification. Patt. Recogn. 44(3) (March 2011) 588-597

30. Allène, C., Audibert, J.Y., Couprie, M., Keriven, R.: Some links between extremum spanning forests, watersheds and min-cuts. IVC 28(10) (2010) 1460-1471 